\title{
Inhibition of mTOR complex 2 restrains tumor angiogenesis in multiple myeloma
}

\author{
Aurelia Lamanuzzi ${ }^{1, *}$, Ilaria Saltarella ${ }^{1, *}$, Vanessa Desantis ${ }^{1}$, Maria Antonia \\ Frassanito ${ }^{2}$, Patrizia Leone ${ }^{1}$, Vito Racanelli ${ }^{1}$, Beatrice Nico ${ }^{3}$, Domenico Ribatti ${ }^{3,4}$, \\ Paolo Ditonno ${ }^{5}$, Marcella Prete ${ }^{1}$, Antonio Giovanni Solimando ${ }^{1}$, Francesco \\ Dammacco ${ }^{1}$, Angelo Vacca ${ }^{1}$ and Roberto Ria ${ }^{1}$ \\ ${ }^{1}$ Department of Biomedical Sciences and Human Oncology, Internal Medicine Unit G. Baccelli, University of Bari Aldo Moro \\ Medical School, Bari, Italy \\ ${ }^{2}$ Department of Biomedical Sciences and Human Oncology, General Pathology Unit, University of Bari Aldo Moro Medical \\ School, Bari, Italy \\ ${ }^{3}$ Department of Basic Medical Sciences, Neurosciences, and Sensory Organs, Section of Human Anatomy and Histology, \\ University of Bari Aldo Moro Medical School, Bari, Italy \\ ${ }^{4}$ National Cancer Institute Giovanni Paolo II, Bari, Italy \\ ${ }^{5}$ Hematology Unit, Di Venere Hospital, Bari, Italy \\ *These authors have contributed equally to this work \\ Correspondence to: Angelo Vacca, email: angelo.vacca@uniba.it \\ Keywords: angiogenesis; endothelial cells; multiple myeloma; mTOR; PP242 \\ Received: June 08, $2017 \quad$ Accepted: March 13, $2018 \quad$ Published: April 17, 2018 \\ Copyright: Lamanuzzi et al. This is an open-access article distributed under the terms of the Creative Commons Attribution License \\ 3.0 (CC BY 3.0), which permits unrestricted use, distribution, and reproduction in any medium, provided the original author and \\ source are credited.
}

\section{ABSTRACT}

The mammalian Target of Rapamycin (mTOR) is an intracellular serine/threonine kinase that mediates intracellular metabolism, cell survival and actin rearrangement. mTOR is made of two independent complexes, mTORC1 and mTORC2, activated by the scaffold proteins RAPTOR and RICTOR, respectively. The activation of mTORC1 triggers protein synthesis and autophagy inhibition, while mTORC2 activation promotes progression, survival, actin reorganization, and drug resistance through AKT hyper-phosphorylation on Ser473. Due to the mTOR pivotal role in the survival of tumor cells, we evaluated its activation in endothelial cells (ECs) from 20 patients with monoclonal gammopathy of undetermined significance (MGUS) and 47 patients with multiple myeloma (MM), and its involvement in angiogenesis. MM-ECs showed a significantly higher expression of mTOR and RICTOR than MGUS-ECs. These data were supported by the higher activation of $\mathrm{mTORC2}$ downstream effectors, suggesting a major role of $\mathbf{m T O R C 2}$ in the angiogenic switch to MM. Specific inhibition of $m$ TOR activity through siRNA targeting RICTOR and dual mTOR inhibitor PP242 reduced the MM-ECs angiogenic functions, including cell migration, chemotaxis, adhesion, invasion, in vitro angiogenesis on Matrige ${ }^{\circledR}$, and cytoskeleton reorganization. In addition, PP242 treatment showed anti-angiogenic effects in vivo in the Chick Chorioallantoic Membrane (CAM) and Matrigel ${ }^{\circledR}$ plug assays. PP242 exhibited a synergistic effect with lenalidomide and bortezomib, suggesting that mTOR inhibition can enhance the antiangiogenic effect of these drugs. Data to be shown indicate that mTORC2 is involved in MM angiogenesis, and suggest that the dual mTOR inhibitor PP242 may be useful for the anti-angiogenic management of MM patients. 


\section{INTRODUCTION}

Multiple myeloma (MM) is a hematologic malignancy characterized by the monoclonal expansion and accumulation of immunoglobulin-secreting plasma cells (PCs) within the bone marrow (BM) [1]. Angiogenesis plays an essential role in MM progression by ensuring tumor growth, PCs invasion and intramedullary/ extramedullary dissemination [2]. In the BM milieu, a correlation is established between microvessel area and the rate of proliferating PCs: angiogenesis is enhanced in patients with active MM compared to those with non-active $\mathrm{MM}$ or with monoclonal gammopathy of undetermined significance (MGUS) [3], and MM PCs themselves trigger angiogenesis that in its turn sustains disease progression.

As emphasized by Hose et al. [4], at variance from memory B cells, normal BMPCs express a surplus of proangiogenic over antiangiogenic genes, resulting in induction of a basal level of in vitro angiogenesis. In MM patients, proangiogenic genes are aberrantly expressed and, conversely, antiangiogenic genes are down-regulated by MMCs, thus explaining the presence of BM angiogenesis to a variable extent in all of them. The accumulation of MMPCs would indeed gradually modify the $\mathrm{BM}$ microenvironment and sustain $\mathrm{BM}$ angiogenesis. Thus, targeting BM angiogenesis is an important therapeutic strategy. However, despite the development of new biologic agents such as bortezomib (the first-inclass proteasome inhibitor) and the immunomodulatory drugs (IMiDs), i.e. thalidomide and lenalidomide with established anti-angiogenic effects [5-7], MM still remains an incurable disease even if the time of relapse is remarkably delayed. Thus, the detection of new drugs, capable of simultaneously targeting tumor cells and angiogenesis, is eagerly awaited.

The Target of Rapamycin (TOR) is a serin-threonine protein kinase belonging to the phosphatidylinositol 3-kinase-related kinases (PIKKs) family [8]. In mammalian cells, TOR is named mammalian Target of Rapamycin (mTOR) that plays a central role in tumor growth, survival and drug resistance. The mTOR forms two different complexes: a) mTOR complex 1 (mTORC1 or $\mathrm{C} 1$ ), that is rapamycin-sensitive and consists of mTOR associated with RAPTOR (regulatory-associated protein of mTOR), mLST8, PRAS40 and Deptor; b) mTOR complex 2 (mTORC2 or $\mathrm{C} 2$ ), that is rapamycin-insensitive and binds RICTOR (rapamycin-insensitive companion of mTOR), mLST8, Sin 1 and Protor. The two complexes play different roles in cellular metabolism: mTORC1 regulates protein translation, promoting cap-dependent mRNA translation through phosphorylation of S6K1 and 4EBP1 (eukaryotic translation initiation factor 4E binding protein-1) [9], and downregulates autophagy through its main target ULK1 [10]. mTORC2 activates serum glucocorticoid-regulated kinase (SGK) and protein kinase $\mathrm{C}$ (PKC) regulating actin cytoskeleton rearrangement, the activities of the small guanosine triphosphatases (GTPases) as RhoA, Cdc42 and Rac1 [11, 12], and phosphorylates its main target protein kinase B (AKT) on Ser473, that promotes cell survival, drug resistance and cell migration $[13,14]$. Thus, the mTORC2 activity may represent a key process in promoting cancer cell invasion and metastases, as well as angiogenesis [14].

Preclinical data with first-generation mTOR inhibitors like rapamycin and rapalogs (such as temsirolimus, everolimus and deforolimus) showed their potential therapeutic activity in MM [15-17], but phase I/ II clinical trials based on these drugs in combination with anti-MM drugs failed [18-20]. Furthermore, MM cell lines and primary MM PCs exhibit both mTORC1 and mTORC2 activation pathways [21]. Although there are currently no specific inhibitors of mTORC2, small molecules such as LY294002, PI-103, and NVP-BEZ235 are able to simultaneously target the adenosine triphosphate (ATP) binding site of both mTOR and PI3K [22, 23]. Accordingly, these inhibitors cannot be used to selectively inhibit mTOR and/or PI3Ks. Feldman et al. reported the synthesis of pyrazolo-pyrimidines (i.e., PP242 and PP30) that are the first dual, selective and ATP-competitive inhibitors of mTOR, with short in vitro half-maximal inhibitory concentrations $\left(\mathrm{IC}_{50}\right.$ values) that are extremely selective for both mTOR complexes [24]. Moreover, PP242 was found to be effective on MM PCs by suppressing both mTORC1 and $\mathrm{mTORC} 2$ activities, thus resulting more effective as anti-cancer agent than rapamycin [25]. Additional data demonstrated higher mTORC2 activation in primary MM PCs and a synergistic effect of PP242 combined with bortezomib, strongly supporting the hypothesis that mTORC2 may be a new therapeutic target in MM [25].

Here, we demonstrate the activation of mTORC2 pathway in BM endothelial cells (ECs) from MM patients (MM-ECs) and its involvement in the regulation of MMECs angiogenesis. We have also addressed the dual mTOR inhibitor PP242 on MM-ECs to assess its anti-angiogenic activity in MM.

\section{RESULTS}

\section{mTOR over-expression and hyper-activation in MM-ECs}

To establish whether mTOR is more activated in MM-ECs than in MGUS-ECs, we examined mTOR mRNA and protein levels in both cells. As shown in Figure 1A, a significantly higher $(+60 \%)$ mTOR mRNA expression was revealed by Real Time RT-PCR in MMECs than MGUS-ECs. When the expression of RAPTOR and RICTOR (that are essential for the activity of mTORC1 and mTORC2, respectively) was assessed, a significant increase of RICTOR was detected in MM-ECs $(+120 \%)$, whereas RAPTOR resulted more expressed in MGUS-ECs $(+80 \%)$. 


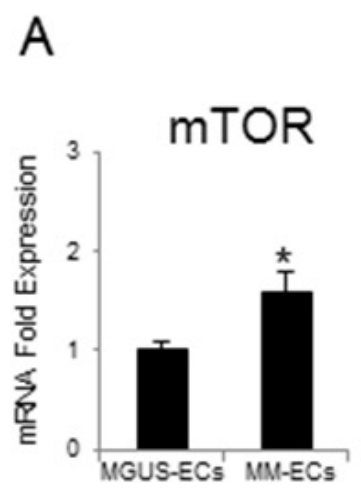

C
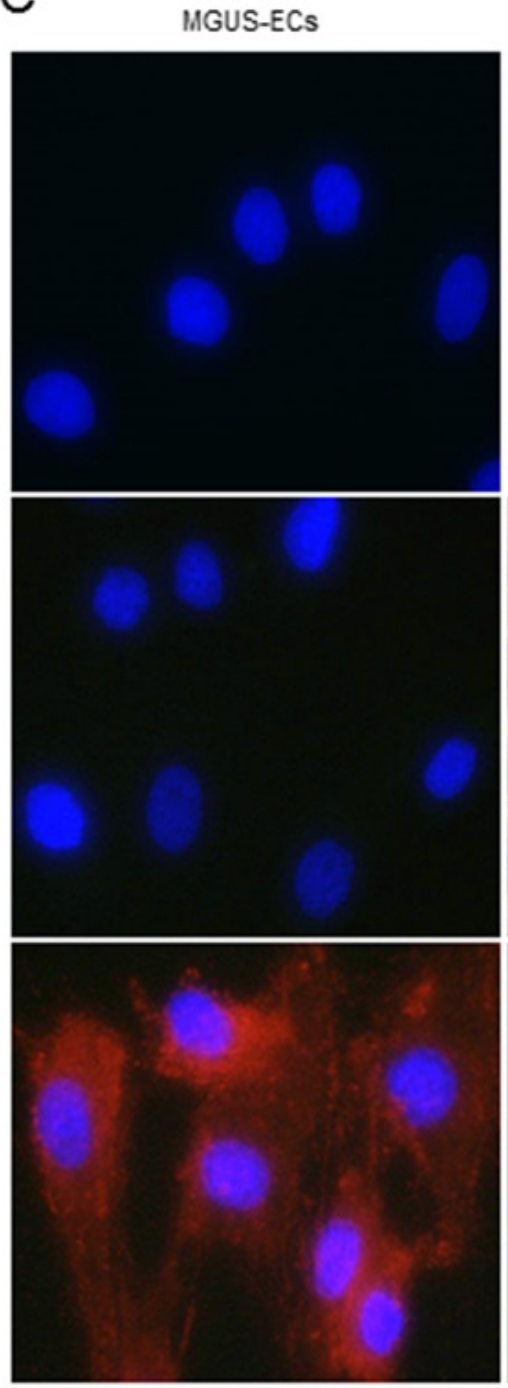
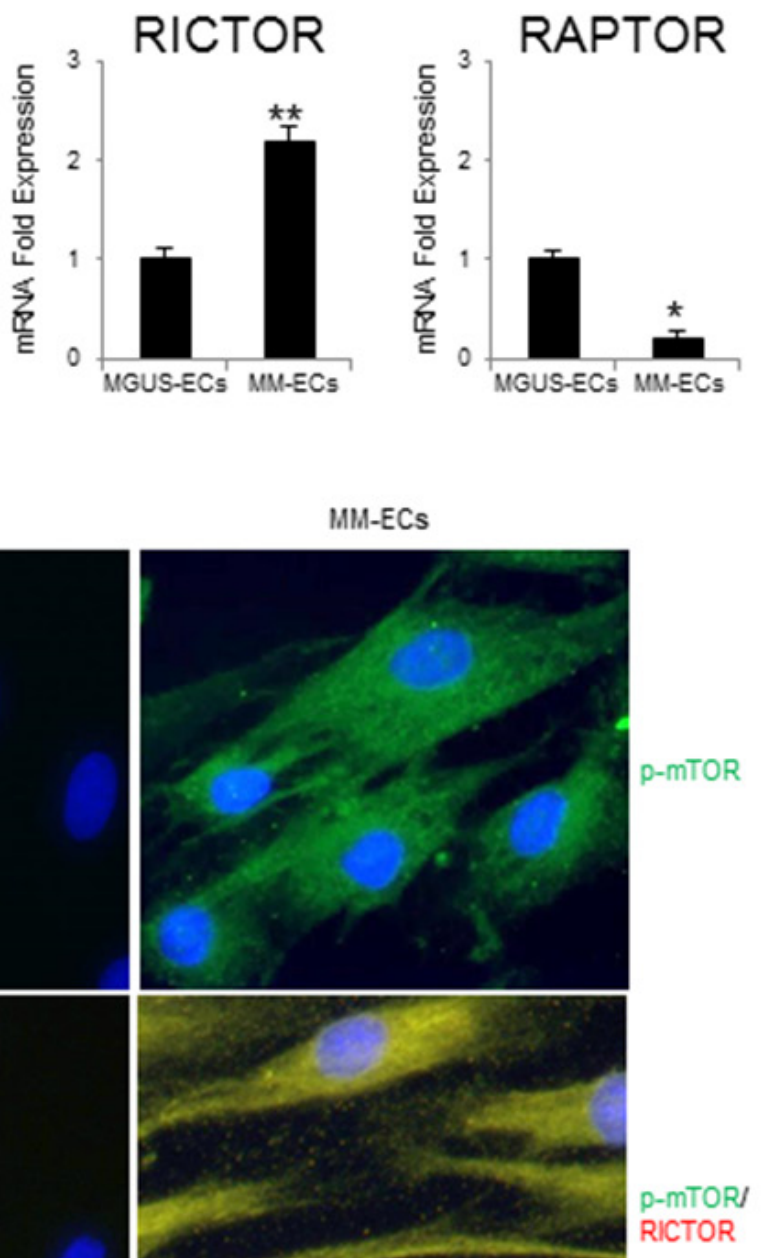

RICTOR
B

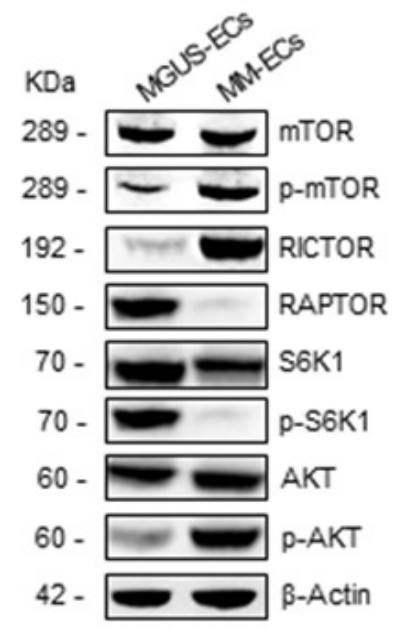

Figure 1: mTOR expression and mTOR activation pathway in MGUS-ECs and MM-ECs. (A) Total mRNA was extracted from MGUS-ECs $(\mathrm{n}=12)$ and MM-ECs $(\mathrm{n}=20)$. mTOR, RICTOR and RAPTOR expression was evaluated by Real-Time RT-PCR and normalized to GAPDH. (B) Total proteins from MGUS-ECs $(\mathrm{n}=12)$ and MM-ECs $(\mathrm{n}=20)$ were analyzed by Western blotting to study mTOR, p-mTOR, RICTOR, RAPTOR, S6K1, p-S6K1, AKT, and p-AKT proteins expression in basal conditions. Immunoreactive bands were normalized to $\beta$-actin. Representative images of single MGUS-ECs and MM-ECs sample are shown. (C) MGUS-ECs and MM-ECs were cultured on chamber-slides, fixed and stained for p-mTOR (green), RICTOR (red) and RAPTOR (red). Nuclei were counterstained with 4',6-diamidino-2-phenylindole (DAPI) (blue). Merge signal (yellow) shows co-localization. Original magnification 400X. Representative images from independent experiments carried out with MGUS-ECs ( $\mathrm{n}=8)$ and MM-ECs $(\mathrm{n}=12)$ are shown. ${ }^{*} \mathrm{p}<0.03$ and ${ }^{* * *} \mathrm{p}<0.003$ by Wilcoxon signed-rank test. 
When mTOR pathway was studied on Western blotting (Figure 1B), mTOR and RICTOR protein levels were found to be increased in MM-ECs but not in MGUSECs. On the contrary, RAPTOR protein was not found in MM-ECs. Moreover, an increased activation of mTOR was detected in MM-ECs in terms of phosphorylation at the Ser2448 [25]. Immunoblotting data highlighted an increase of total AKT (mTORC2 target) in MM-ECs, and a significant increase of its activation ( $p-S e r 473)$. Instead, the main mTORC1 target S6K1 was more activated (p-Thr389) in MGUS-ECs than in MM-ECs. This was confirmed by immunofluorescence (Figure 1C): in MMECs, p-mTOR (green) co-localized with RICTOR (red), but not with RAPTOR (red).

These results indicate that $\mathrm{mTORC} 2$ pathway is more activated in MM-ECs than in MGUS-ECs and that it is likely involved in the regulation of MM-ECs activities.

\section{Effects of RICTOR knockdown in MM-ECs}

To investigate whether mTORC2 is directly involved in MM angiogenesis, several functional assays using MM-ECs with RICTOR knocked-down were carried out. RICTOR silencing was performed through the siRNA transfection. By Real-Time RT-PCR and Western blotting, we assessed the optimal time and concentration of siRICTOR to ensure its inhibition. Supplementary Figure 1 shows that in MM-ECs treated with siRICTOR (25 nM for $72 \mathrm{~h}$ ) the RICTOR expression was strikingly reduced (-75\% at both mRNA and protein levels). Immunoblotting analysis also revealed a significant reduction of both $\mathrm{p}-\mathrm{AKT}$ and p-mTOR proteins in MM-ECs silenced for 72h (Supplementary Figure 1C).

Figure 2A shows that, different from control cells whose intracellular Phalloidin-stained actin fibers appeared continuous and well-distributed, siRICTOR MM-ECs gave these fibers sparse and fragmented, indicating that RICTOR silencing interferes with actin reorganization. Also, compared to control cells that were able to fully repair the scratch, siRICTOR transfection reduced MM-ECs ability to repair a wound (-80\%) (Figure $2 \mathrm{~B})$, to migrate in response to a chemical gradient of chemo-attractive agents, as shown by chemotaxis assay $(-52 \%)$ (Figure 2C), to invade extra-cellular matrix (ECM; $-92 \%$ ) (Figure 2D) and to adhere to fibronectin $(-75 \%)$ (Figure 2E).

Finally, control MM-ECs transfected with random siRNAs actively produced angiogenesis in vitro, based on a complex organization in which cells communicate to each other through the formation of a network with many junctions and branching points. On the contrary, MM-ECs transfected with siRICTOR were unable to form these networks. The capillaries on Matrigel ${ }^{\circledR}$ were scanty and disorganized with few junctions, in the absence of empty area formation, as shown by the significant reduction of mesh area $(-87 \%)$, branching points $(-73 \%)$, and vessel length $(-72 \%)$ (Figure $2 \mathrm{~F})$.
Experiments of RAPTOR knockdown were also performed, but results were not significant due to the low levels of RAPTOR in MM-ECs.

\section{Effects of PP242 treatment on MM-ECs}

We next investigated whether the use of the dual mTOR inhibitor PP242 might have the same effects of RICTOR knockdown in MM-ECs. To find out the PP242 concentration with the highest inhibition of the functional abilities of MM-ECs, the wound-healing assay was performed with increasing concentrations of PP242. As shown in Supplementary Figure 2A, after treatment with PP242 at 10, 50, 100 and $200 \mathrm{nM}$, the mobility of MM-ECs was gradually reduced in step with the increased drug concentration. No effect on MM-ECs apoptosis and proliferation was observed (Supplementary Figure 2B and 2C). Western blot analysis of MM-ECs treated with PP242 (100 nM for 48h) showed a remarkable reduction of p-mTOR and p-AKT and a not significant effect on p-S6K1 and p-ERK1/2 (Supplementary Figure 2D), indicating that PP242 inhibits the mTOR pathway.

Next, we evaluated the effect of PP242 on MM-ECs angiogenesis by Matrigel ${ }^{\circledR}$ assay. Results were compared to those obtained with the $\mathrm{mTORC} 1$ inhibitor rapamycin (Figure 3). PP242 treatment reduced the constitutive MM-ECs capability to form capillary-like structures on Matrigel $^{\circledR}$ as shown by mesh area $(-80 \%)$, vessel length $(-70 \%)$ and branching points $(-58 \%)$ inhibition, corroborating the role of $\mathrm{mTORC} 2$ in MM angiogenesis. By contrast, treatment of MM-ECs with rapamycin (5 nM for 48h) did not affect angiogenesis in vitro in line with the low expression of mTORC1 in MM-ECs.

PP242 was able to alter actin organization, as revealed by immunofluorescence; at variance from controls, in MM-ECs treated with PP242 the F-actin appeared fragmented with gaps (Figure 4A). Moreover, PP242 reduced MM-ECs motility, as demonstrated by the reduction of spontaneous migration (-70\%) (Figure 4B) and of chemotaxis ability (-40\%) (Figure 4C). Given that the chemo-invasive property of treated MM-ECs was also decreased (-86\%) (Figure 4D), we investigated whether the treatment with the PP242 was able to alter the secretion of metalloproteinases (MMPs) using the zymography assay. As shown in Figure 4E, the secretion of the inactive precursor of MMP-2 (pro-MMP-2) and the active form of MMP-2 were significantly reduced ( $-40 \%$ and $-60 \%$, respectively) in treated MM-ECs compared to controls. The adhesion assay also revealed a significantly reduced ability $(-52 \%)$ of treated MM-ECs to adhere to fibronectin (Figure 4F).

\section{Effects of PP242 on angiogenesis in vivo}

Preliminarily, we investigate the PP242 effects on the release of angiogenic cytokines by the Q-Plex assay. This specifically analyses the following pro-angiogenic 


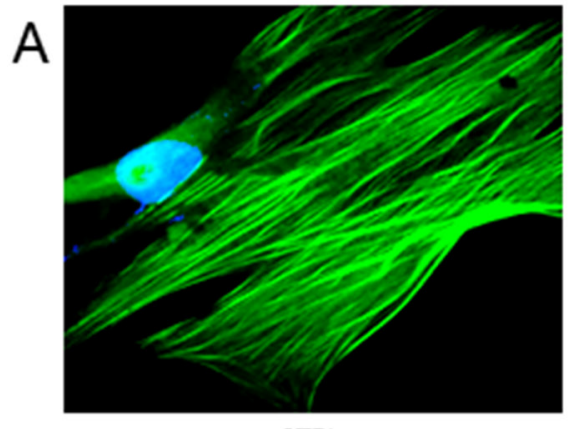

CTRL

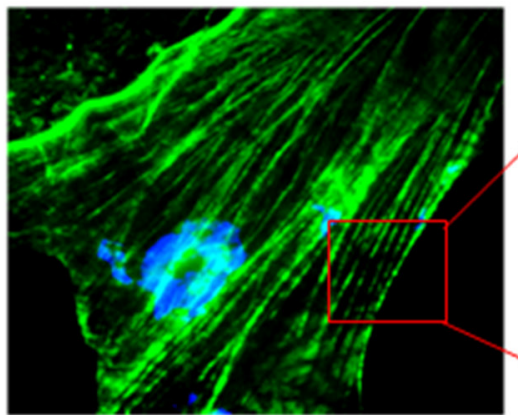

siRICTOR

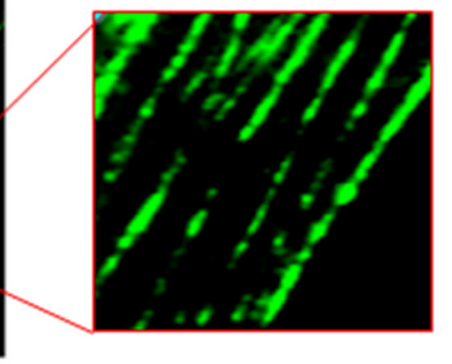

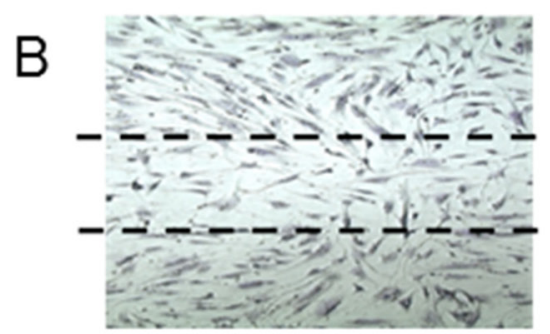

CTRL

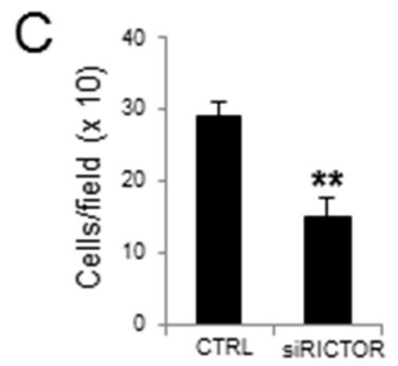

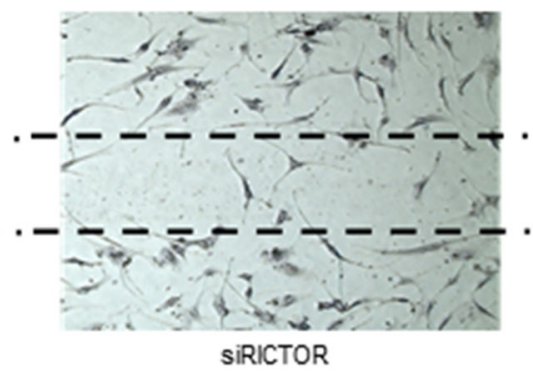

D

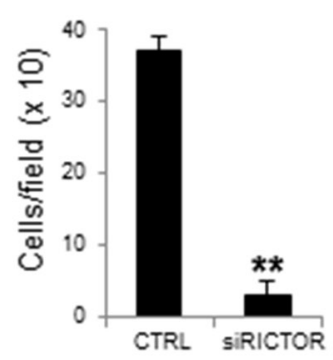

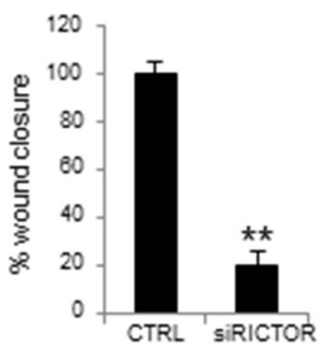

$\mathrm{E}$

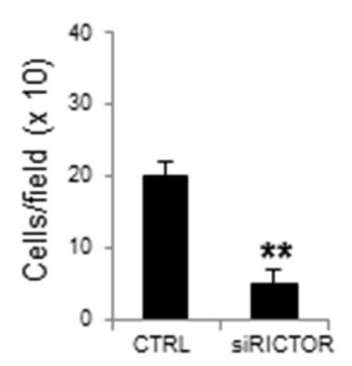

$\mathrm{F}$
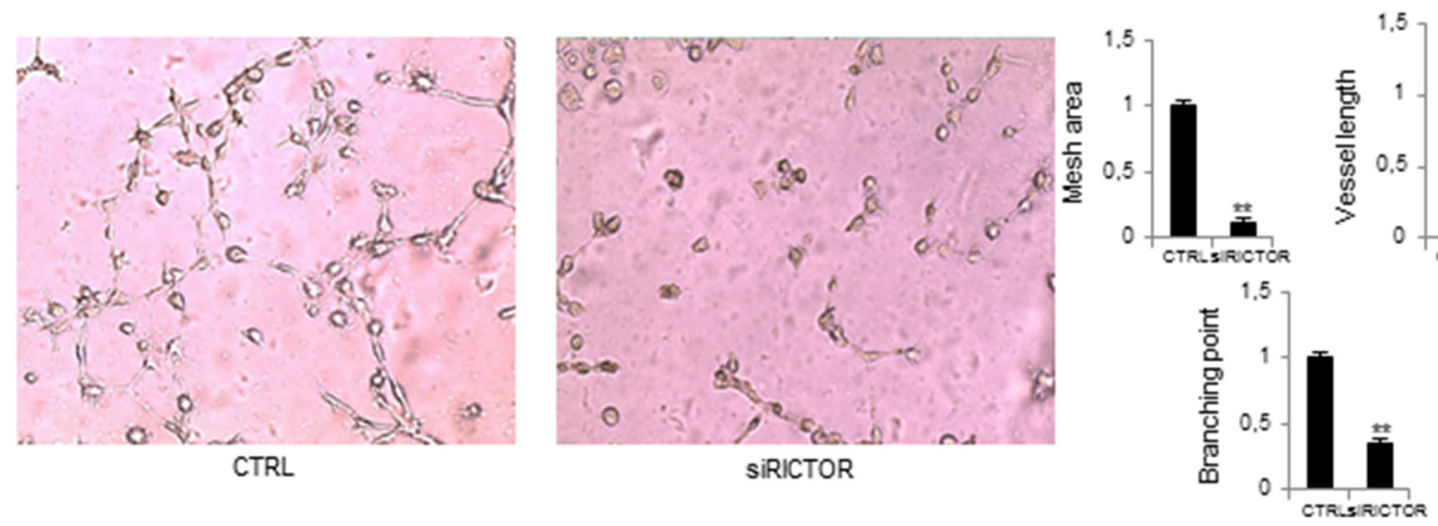

Figure 2: Effects of RICTOR knockdown on MM-ECs. MM-ECs ( $\mathrm{n}=8$ ) were treated with scramble siRNA or with RICTOR siRNA ( $25 \mathrm{nM}$ for $72 \mathrm{~h}$ ) and tested for (A) immunofluorescence to stain actin fibers with Phalloidin (green). Nuclei were counterstained with 4',6-diamidino-2-phenylindole (DAPI) (blue). Original magnification 400X; (B) spontaneous migration to repair the scratch in the wound healing. Original magnification $200 \mathrm{X}$. Data are expressed as mean $\pm \mathrm{SD} ;(\mathbf{C})$ chemotaxis migration in response to chemoattractant agents $(10 \mathrm{ng} / \mathrm{mL}$ FGF-2, $10 \mathrm{ng} / \mathrm{mL}$ VEGF and 1.5\% FBS) in Boyden chamber. Data are expressed as mean $\pm \mathrm{SD}$; (D) chemoinvasion in response to chemoattractant agents $\left(10 \mathrm{ng} / \mathrm{mL}\right.$ FGF-2, $10 \mathrm{ng} / \mathrm{mL}$ VEGF and 1.5\% FBS) through Matrigel ${ }^{\circledR}$ matrix. Data are expressed as mean $\pm \mathrm{SD}$; (E) adhesion to fibronectin-coated 96-well plates of MM-ECs stained with Calcein AM. Data are expressed as mean $\pm \mathrm{SD}$; (F) in vitro angiogenesis on Matrige $\mathrm{l}^{\circledR}$ matrix-coated 48-well plates. Representative images from 8 independent experiments are shown. Original magnification 200X. Bar graphs indicate relative mesh area, vessel length and branching points, analysed by EVOS software. Data are expressed as mean \pm SD. ${ }^{* *} \mathrm{p}<0.003$ by Wilcoxon signed-rank test. 
cytokines: angiopoietin-2 (ANG-2), hepatocyte growth factor (HGF), interleukin-8 (IL-8), tumor necrosis factor- $\alpha$ (TNF- $\alpha$ ), fibroblast growth factor-2 (FGF-2), plateletderived growth factor (PDGF) and vascular endothelial growth factor (VEGF), and the anti-angiogenic molecules tissue inhibitor of metalloproteinase (TIMP) -1 and -2 . Analysis of conditioned medium (CM) from MM-ECs, treated or not with PP242, showed a significant reduction of ANG-2, FGF-2, HGF, PDGF and VEGF released after treatment (Figure 5A).

We then asked the question whether PP242 was also able to inhibit in vivo angiogenesis. To this end, we performed the Chick chorioallantoic membrane (CAM) assay and the Matrigel ${ }^{\circledR}$ plug assay. Figure 5B shows that CM from MM-ECs pre-treated with PP242 (100 nM for $48 \mathrm{~h}$ ) significantly inhibited in vivo angiogenesis: the number of vessels was $9 \pm 3$ for CM from treated MM-ECs and $28 \pm 4$ for untreated cells. Similar data were obtained testing CM from MM-ECs treated with PP242 for 24h that resulted in the formation of $11 \pm 2$ vessels converging toward the sponge (data not shown). When we tested the direct effect of MM-ECs pre-treated with PP242 for 48h in the in vivo CAM assay (Figure 5C), we counted $27 \pm 5$ vessels for control MM-ECs, while $12 \pm 3$ for pre-treated
MM-ECs. Finally, the anti-angiogenic effect of PP242 was analysed in NOD/SCID mice by Matrigel ${ }^{\circledR}$ plug assay. Injection of CM from control MM-ECs attracted the mice CD31+ ECs into the plug and induced angiogenesis: plugs were macroscopically highly vascularized and appeared red colored (Figure 5F) in much the same way as in mice injected with Matrigel ${ }^{\circledR}$ supplemented with FGF-2 and VEGF (positive control) (Figure 5E). Immunofluorescence analysis of $\mathrm{CD} 31+$ cells highlighted the organization of mice ECs into vessels (Figure 5E and 5F). CM from PP242-treated MM-ECs did not show the capability to attract $\mathrm{CD} 31+$ cells into plugs that were less colored (Figure 5G) in much the same way as in mice injected with Matrigel ${ }^{\circledR}$ added with SFM (negative control) (Figure 5D). Furthermore, PP242 treatment of mice injected with Matrige ${ }^{\circledR}$ added with FGF-2 and VEGF (Figure 5I) showed that PP242 treatment inhibited migration of mice ECs and, thus, angiogenesis in vivo compared to vehicletreated mice (Figure $5 \mathrm{H}$ ).

\section{PP242 acts in synergy with anti-myeloma drugs}

Finally, we assessed the ability of PP242 inhibitor to act in synergy with two anti-myeloma and anti-angiogenic

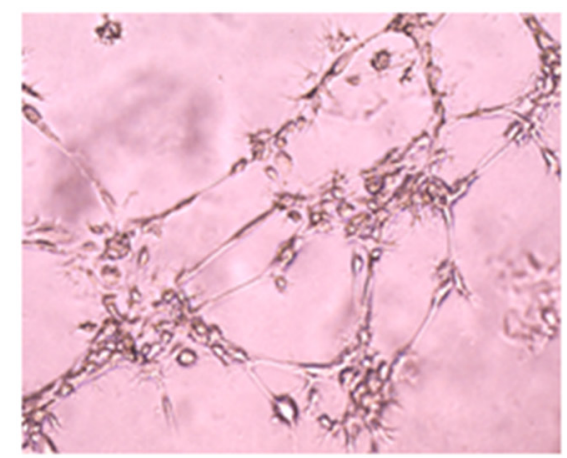

CTRL

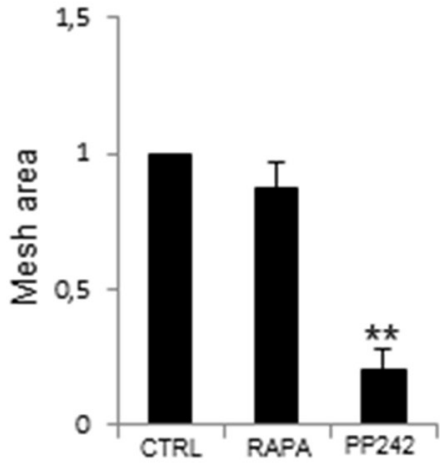

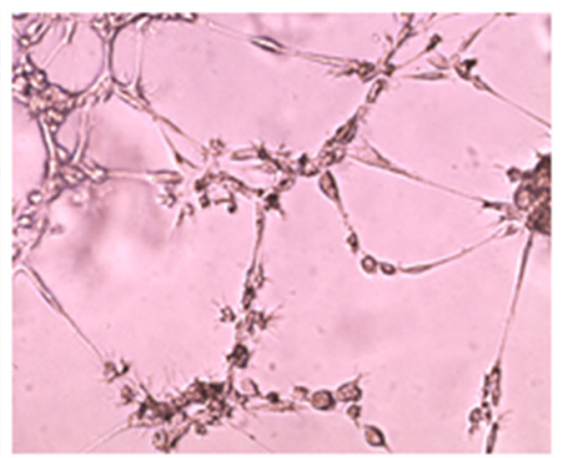

RAPA

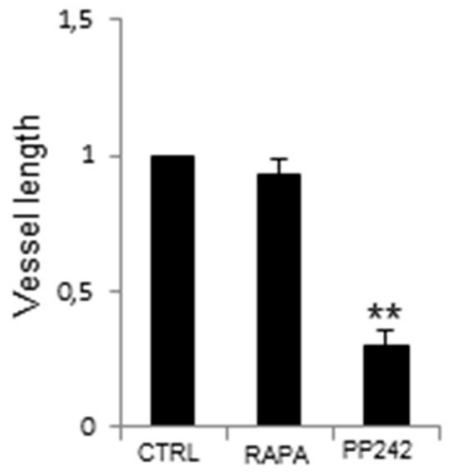

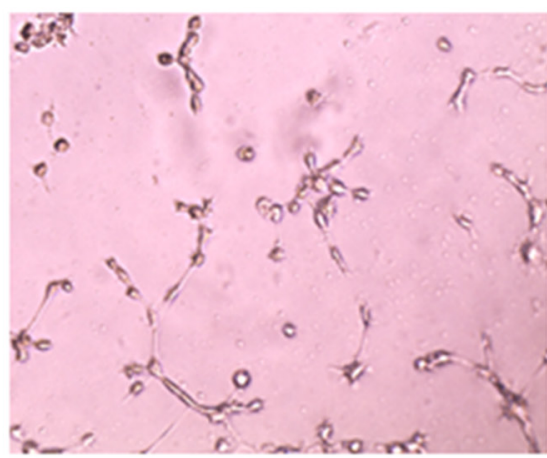

PP242

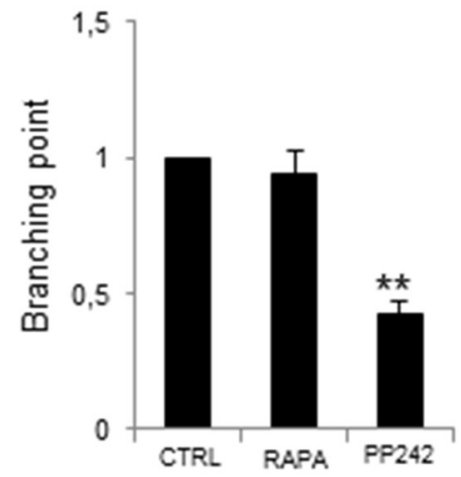

Figure 3: PP242 inhibition of MM-ECs angiogenesis in vitro. MM-ECs $(\mathrm{n}=6)$ were treated with rapamycin (5 $\mathrm{nM}$ for $48 \mathrm{~h})$ or PP242 (100 nM for 48h) or untreated (CTRL) and tested for in vitro angiogenesis on Matrigel ${ }^{\circledR}$ assay. Original magnification $200 \mathrm{X}$. Representative images of 6 independent experiments are shown. Bar graphs indicate relative mesh area, vessel length and branching points analysed by EVOS software. Data are expressed as mean \pm SD. ${ }^{* *} \mathrm{p}<0.003$ by Wilcoxon signed-rank test. 
drugs, such as bortezomib [4] and lenalidomide [7]. The synergistic effects of PP242 with anti-MM drugs were tested by in vitro angiogenesis on Matrigel ${ }^{\circledR}$ assay (Figure 6). PP242, bortezomib and lenalidomide alone were able to disrupt the network formation of capillaries on the Matrigel ${ }^{\circledR}$ surface, compared to control MM-ECs. Interestingly, PP242 acted in synergy with anti-MM drugs: MM-ECs treated with PP242 in conjunction with bortezomib or lenalidomide showed a greater reduction of branches and capillary formation on Matrigel ${ }^{\circledR}$ matrix compared to PP242, or bortezomib or lenalidomide alone, as demonstrated by the significant reduction of mesh area, vessel length and branching points.

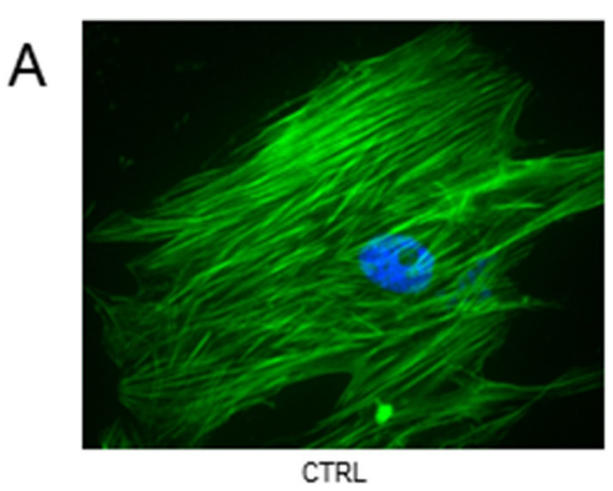

CTRL
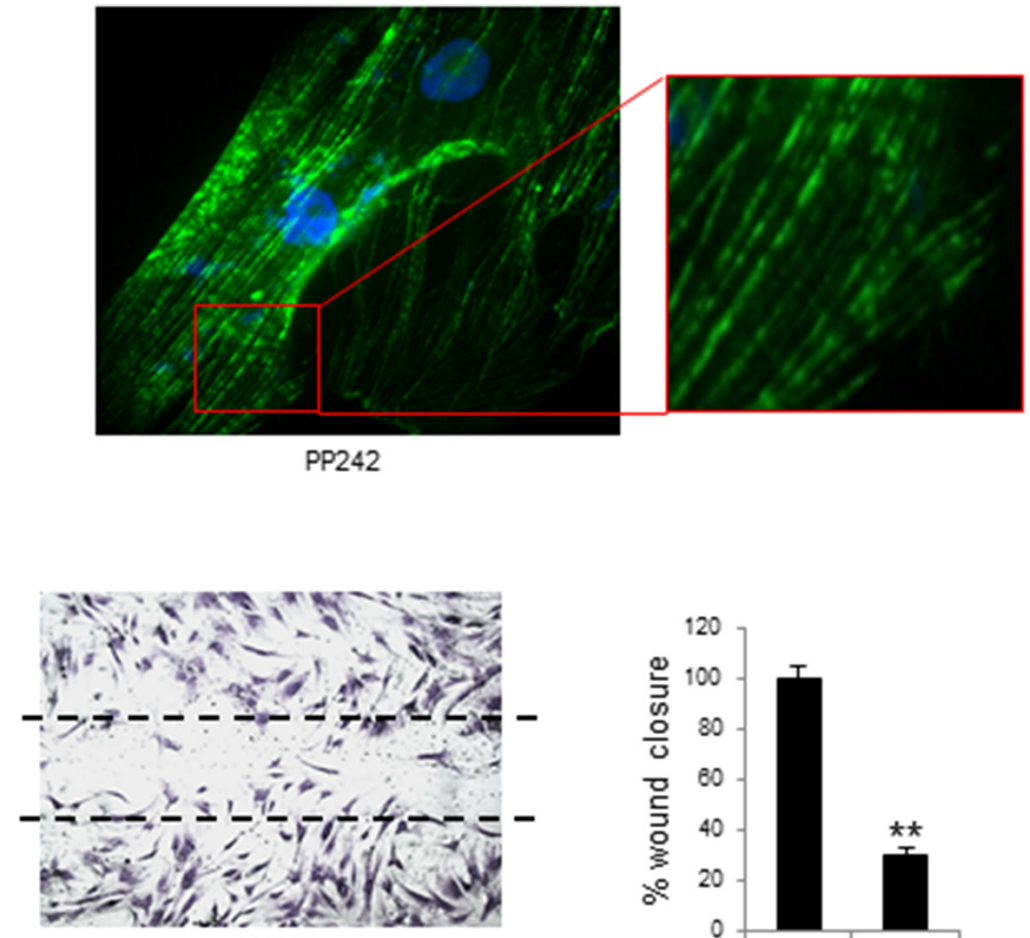

PP242

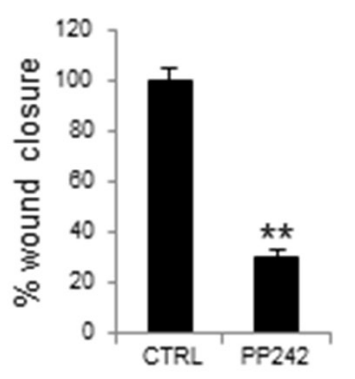

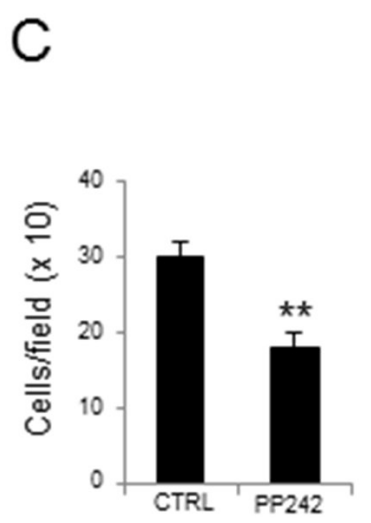
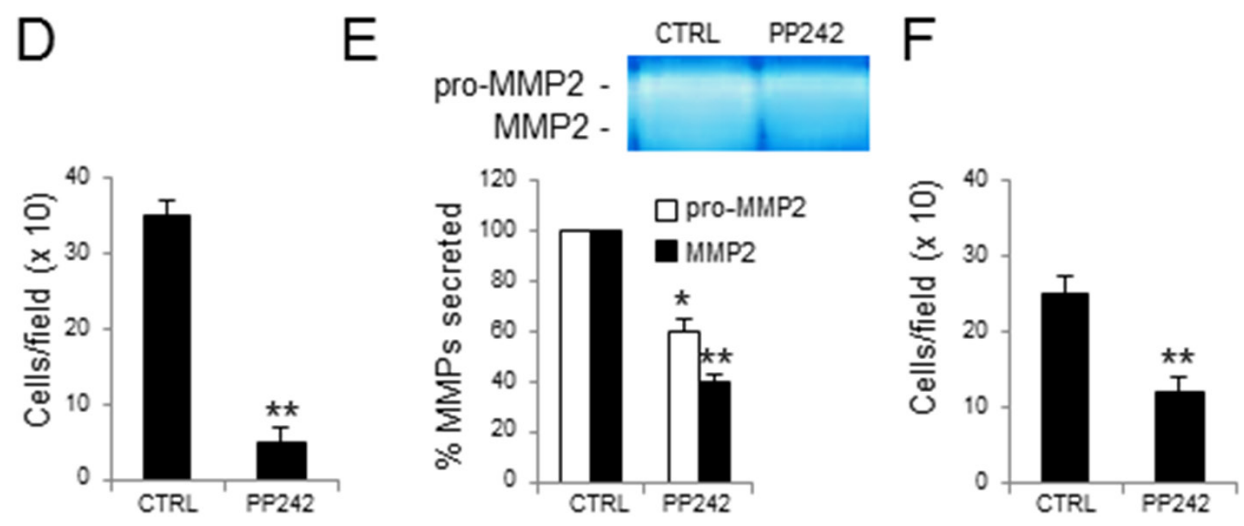

Figure 4: Effects of PP242 treatment on MM-ECs. MM-ECs (n=10), treated or not with PP242 (100 nM for 48h), were tested for (A) immunofluorescence to stain actin fibers with Phalloidin (green), nuclei were counterstained with 4',6-diamidino-2-phenylindole (DAPI) (blue). Original magnification 400X; (B) spontaneous migration to repair the scratch in the wound healing. Original magnification 200X. Data are expressed as mean $\pm \mathrm{SD}$; (C) chemotaxis in response to chemoattractant agents $(10 \mathrm{ng} / \mathrm{mL} \mathrm{FGF}-2,10 \mathrm{ng} / \mathrm{mL} \mathrm{VEGF}$ and $1.5 \%$ FBS) in Boyden chamber. Data are expressed as mean $\pm \mathrm{SD}$; (D) chemoinvasion in response to chemoattractant agents (10 ng/mL FGF-2, $10 \mathrm{ng} / \mathrm{mL}$ VEGF and 1.5\% FBS) through Matrigel ${ }^{\mathbb{B}}$ matrix. Data are expressed as mean \pm SD; (E) zymography of CM to determine the amount of active and inactive form of MMP2. Region of protease activity appeared as white bands and the percentage of MMPs release was estimated using Gel Logic 1,500 Imaging System. Data are expressed as mean \pm SD; (F) adhesion to fibronectin-coated 96-well plates of MM-ECs stained with Calcein AM. Data are expressed as mean \pm SD. ${ }^{*} \mathrm{p}<0.03$ and ${ }^{* *} \mathrm{p}<0.003$ by Wilcoxon signed-rank test. 
A

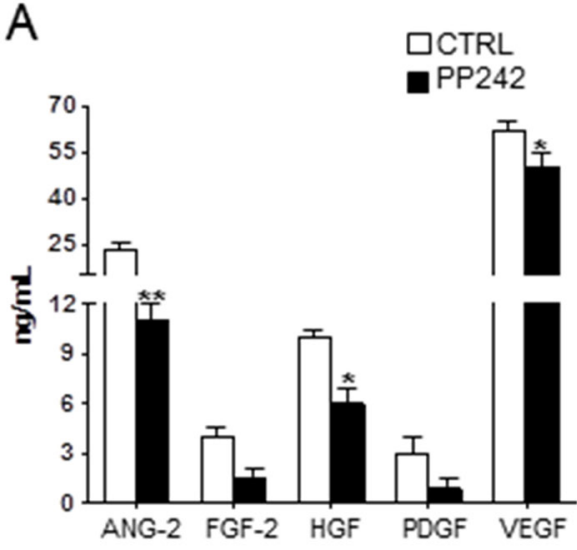

B
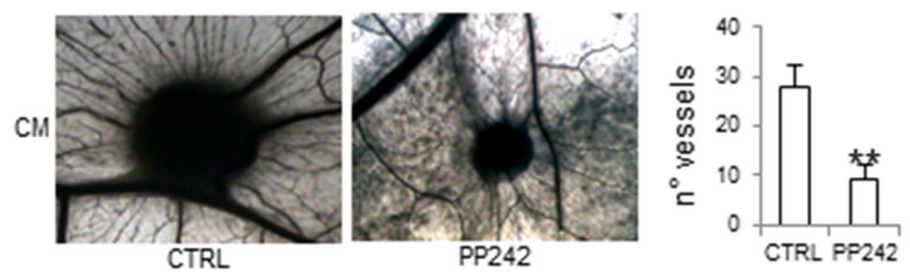

C
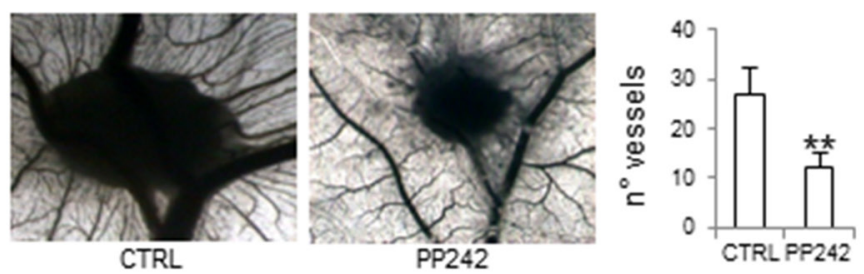

D

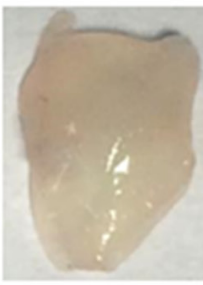

$\mathrm{F}$

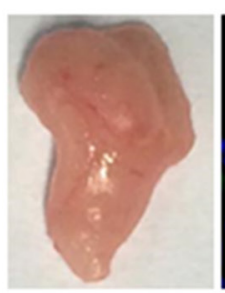

SFIM

(CTRL neg)

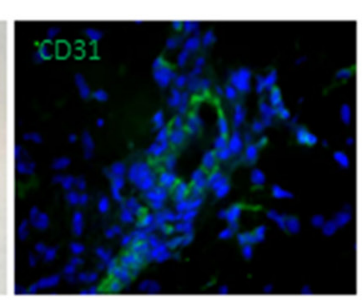

CM CTRL

$\mathrm{H}$

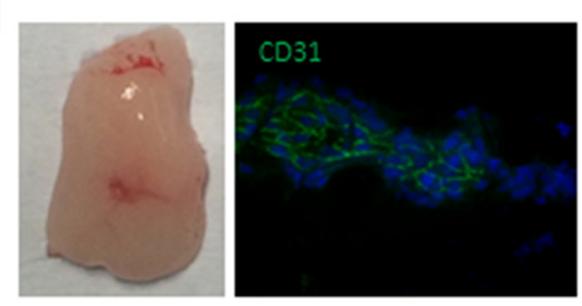

CTRL pos+DIMSO
E

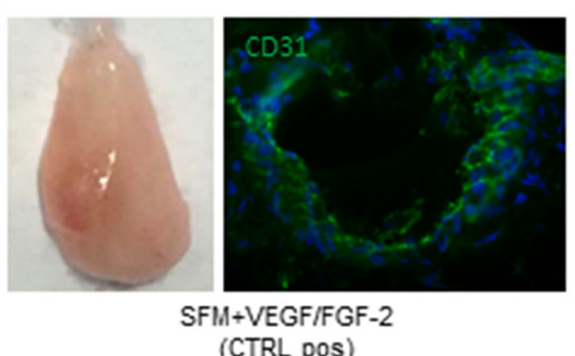

G
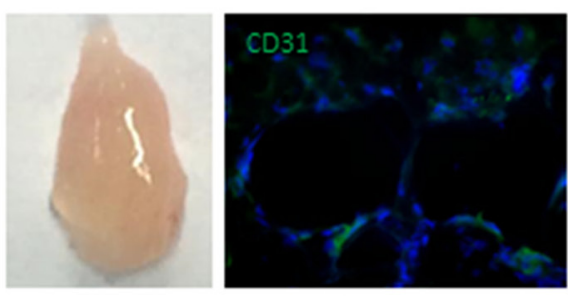

CM PP242

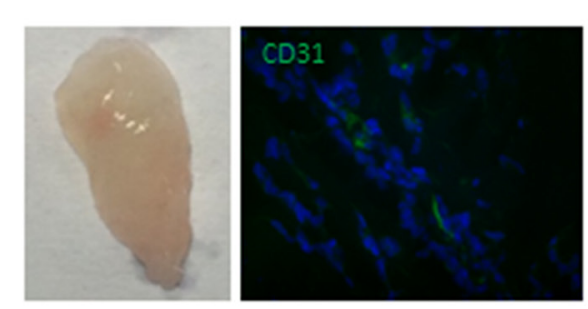

CTRL pos+PP242

Figure 5: Effects of PP242 on angiogenesis in vivo. Conditioned media (CM) from MM-ECs ( $\mathrm{n}=16)$ treated or not with PP242 (100 nM for 48h) were tested for (A) pro-angiogenic cytokine release in a multiplex ELISA assay and (B) CAM assay. (C) CAM assay with MM-ECs ( $\mathrm{n}=5$ ) pre-treated or not with PP242 (100 nM for 48h). Original magnification 50X. Representative images from 5 independent experiments are shown. (D-I) Matrigel ${ }^{\circledR}$ plug assay and immunofluorescence analysis of mouse CD31+ cells (green) from plug sections. NOD/SCID mice were injected with Matrigel ${ }^{\circledR}$ containing: (D) serum free medium (SFM) as negative control; (E) SFM supplemented with VEGF/FGF-2 as positive control; (F) CM from untreated MM-ECs (n=4); (G) CM from MM-ECs (n=4) treated with PP242 (100 nM for 48h). (H-I) NOD/SCID mice injected with Matrige ${ }^{\circledR}$ containing SFM+VEGF/FGF-2 were intraperitoneally treated with (H) DMSO-vehicle or (I) PP242. Original magnification 200X. Representative images from 4 independent experiments are shown. ${ }^{*} \mathrm{p}<0.03$ and ${ }^{* *} \mathrm{p}<0.003$ by Wilcoxon signed-rank test. 


\section{DISCUSSION}

The role of mTOR pathway in tumor angiogenesis has been documented and associated to the activity of mTORC1 complex [26-28]. Indeed, the blockage of mTOR with rapamycin and rapalogs reduces tumor angiogenesis by inhibiting ECs functions and VEGF secretion [29]. Frost et al. demonstrated that the antitumor effect of rapamycin inhibitors in vivo is due to the down-regulation of VEGF secretion and the subsequent inhibition of angiogenesis [30].

Here we demonstrated that the activation of mTORC2 pathway is an important pro-angiogenic feature of MM-ECs. Our data highlighted an increase of mTOR phosphorylation on S2884, associated to a

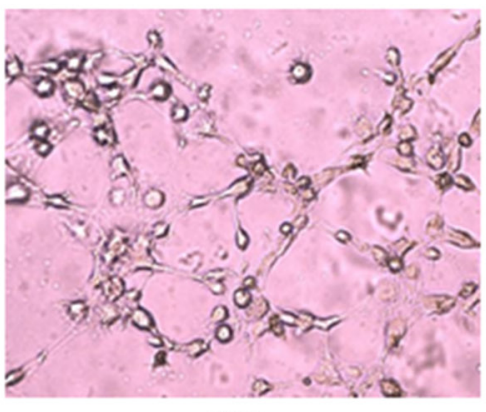

CTRL

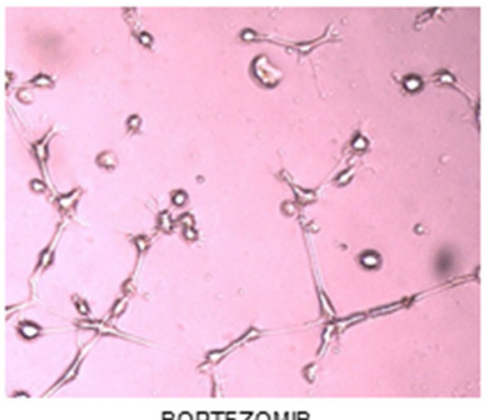

BORTEZOMIB

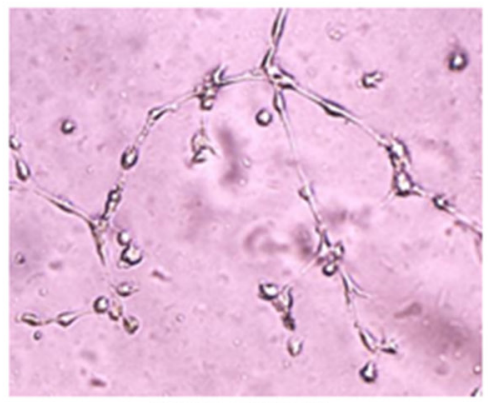

LENALIDOMIDE
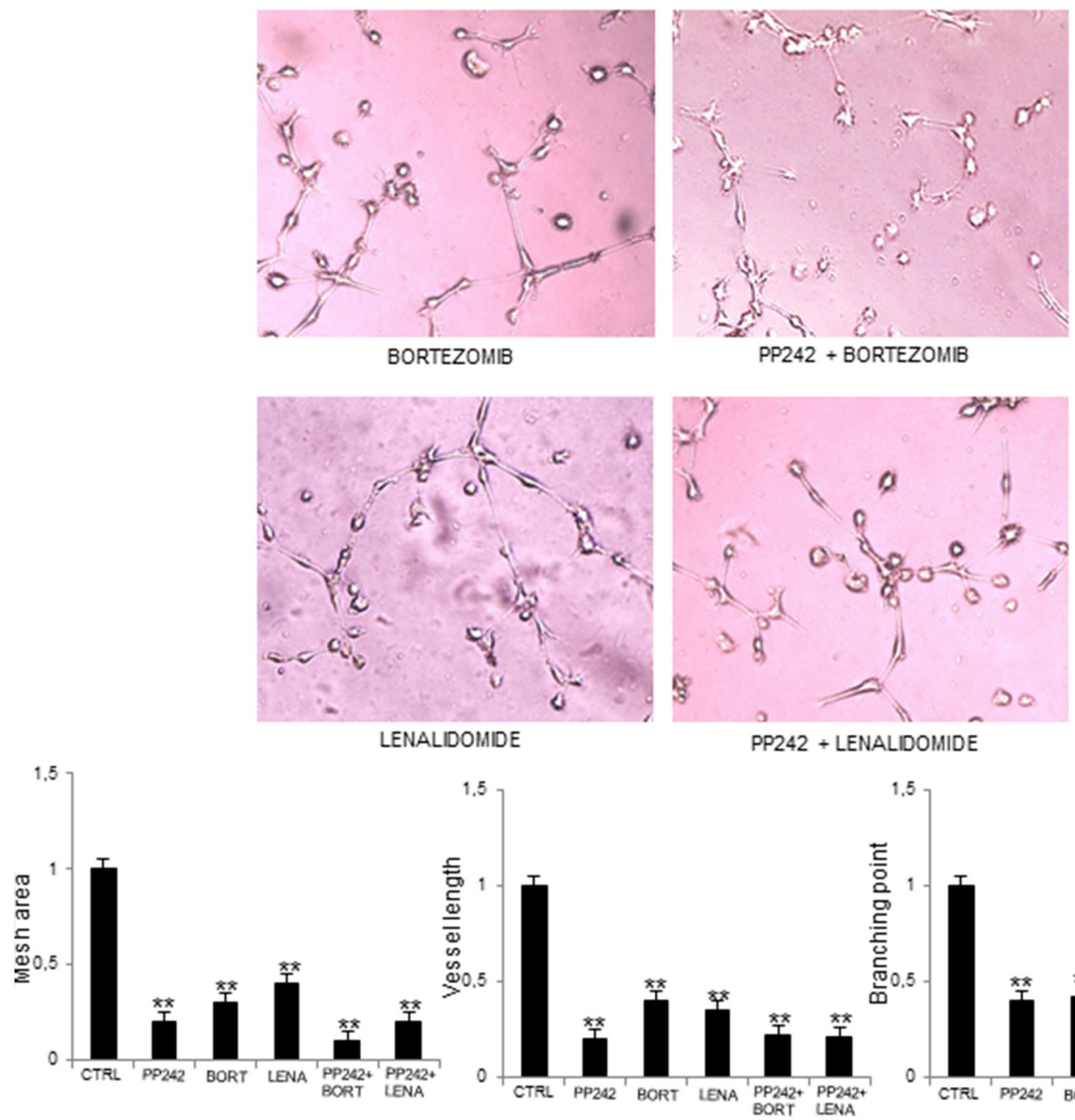

PP242 + BORTEZOMIB

DRT LENA

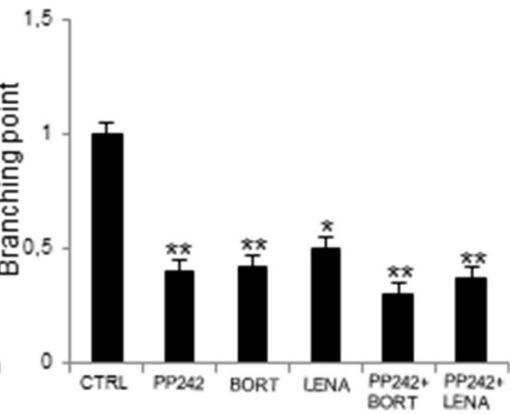

Figure 6: PP242 acts in synergy with other anti-myeloma drugs. MM-ECs ( $\mathrm{n}=10)$ were untreated or treated with PP242 (100 $\mathrm{nM})$, bortezomib $(7.5 \mathrm{nM})$ or lenalidomide $(1.75 \mu \mathrm{M})$, alone or in combination, for $48 \mathrm{~h}$ and tested for in vitro angiogenesis assay. Original magnification 200X. Bar graphs indicate relative mesh area, vessel length and branching points, analysed by EVOS software. Data are expressed as mean \pm SD. ${ }^{*} \mathrm{p}<0.03$ and ${ }^{* *} \mathrm{p}<0.003$ by Wilcoxon signed-rank test. 
higher expression of RICTOR and to a rise in AKT phosphorylation on Ser473 in MM-ECs, indicating that mTORC2 is more activated than mTORC1 [12]. On the contrary, MGUS-ECs showed an increase of S6K1 phosphorylation as sign of mTORC1 activation, suggesting a shift from one complex to the other during the progression from MGUS to MM. RICTOR knockdown decreased MM-ECs cytoskeletal reorganization, migration, chemo-invasion, adhesion and in vitro angiogenesis on Matrige ${ }^{\circledR}$ assay, i.e., all cell functions needed for angiogenesis to develop in $\mathrm{MM}[31,32]$.

In spite of mTOR crucial role in tumor progression, the first generation of mTOR inhibitors (rapamycin and rapalogs) have shown limited effects in phase I/II clinical trials in solid tumors [33-36]. In MM, clinical trials based on rapalogs in combination with anti-myeloma drugs have shown low activity [17-19], plausibly due to a negative feedback loop between mTORC1 and mTORC2 [13]. Inhibition of $\mathrm{mTORC} 1$ by rapamycin results in the hyperactivation of p-AKT pathway, by the stimulation of mTORC 2 and, consequently in enhanced survival and chemoresistance of MM-PCs [13, 20, 37]. Based on these observations, mTOR inhibitors blocking both mTORC1/2 complexes have been developed. Promising preclinical studies showed a greater anti-proliferative and pro-apoptotic effect of the dual mTORC1/2 inhibitors compared to rapamycin. Some of these compounds, i.e. INK-128, MLN0128, TAK-228 are in phase I/II of clinical trials in advanced solid tumors, lymphomas as well as in MM [38, 39].

Given that MM-ECs show activated mTORC2 signaling and are insensitive to rapamycin, we wondered whether PP242 is a possible anti-angiogenic drug in MM. PP242 is a pyrazolo-pyrimidine belonging to the class of dual mTOR inhibitors that are able to block specifically mTORC1 and mTORC2 [24]. PP242 treatment at noncytotoxic concentration inhibited angiogenesis both in vitro and in vivo. Accordingly, to the regulation of Rho and cytoskeleton reorganization mediated by mTORC2 [40, 41], PP242 alters actin reorganization that affects migration, chemotaxis, chemoinvasion and adhesion of MM-ECs. Reduction of cell adhesion on fibronectin was not due to a down-regulation in the expression of the $\alpha_{\mathrm{v}} \beta_{3}$ (data not shown), which is the most expressed integrin by MMECs $[42,43]$. Perhaps the alteration of F-actin fibers, that interact with the $\mathrm{C}$-terminal region of $\beta$-integrins favoring their integrity and functions [44], affects the interplay with $\alpha_{v} \beta_{3}$ integrin and, as a consequence, its activity.

Activation of the PI3K/AKT/mTOR pathway regulates the release of VEGF and of others proangiogenic factors, i.e. nitric oxide, angiopoietins [45] and of MMP-2 and MMP-9 [46]. Accordingly, the PP242 treatment significantly reduced the secretion of ANG-2, VEGF, FGF-2, HGF and of pro-MMP-2 and active MMP-2. Both VEGF and HGF are implicated in $\mathrm{MM}$ angiogenesis due to the existence of autocrine and paracrine loops in MM that further activate MM-ECs
[47, 48]. ANG-2 promotes ECs proliferation, migration, sprouting, and neovascularization in response to VEGF [49-51], and its inhibition reduces tumor angiogenesis. In addition, the inhibition of MMP-2 release along with the interference on cytoskeleton rearrangement contributes to the decreased MM-ECs chemoinvasion.

According to the reduction of release of proangiogenic cytokines, the CM from PP242-treated MMECs was unable to induce angiogenesis in the in vivo $\mathrm{CAM}$ and Matrigel ${ }^{\circledR}$ plug assays. Conversely, CM from untreated MM-ECs induced vessel formation in both in vivo assays. Furthermore, the anti-angiogenic effect of the drug on MM-ECs angiogenic activities, i.e. migration, chemotaxis and angiogenesis, was confirmed in vivo by intraperitoneally treated NOD/SCID mice with PP242. Taken together, the in vitro and in vivo results highlight the importance of $\mathrm{mTORC} 2$ in the regulation of MM angiogenesis suggesting the PP242 as a new possible antiangiogenic molecule in $\mathrm{MM}$.

In line with our study, by using RAPTOR and/ or RICTOR knockdown mouse models, Wang et al. demonstrated the involvement of mTORC2 in the regulation of ECs proliferation and angiogenesis both in vitro and in vivo, whereas the loss of function of MTORC1 gave only marginal effect on the ECs activities [12]. Hoang et al. have further shown that PP242 is able to exert antiMM effects on several MM cell lines as well as on primary PCs from three newly diagnosed MM patients [25].

The important role of the relationship between angiogenesis and PCs in MM pathogenesis and progression is well known $[4,47,48,52,53]$. The new anti-myeloma therapeutic approaches, i.e. bortezomib [5] and IMiDS, such as thalidomide [54] and lenalidomide [7], that target both BM PCs and angiogenesis, have been well established in MM.

In conclusion, mTORC2 is mainly involved in the pro-angiogenic properties of MM-ECs. The selective inactivation of mTORC2 on MM-ECs through RICTOR silencing, and dual mTOR inhibition by PP242 restrains angiogenesis both in vitro and in vivo. Therefore, PP242 seems to be a promising agent with direct anti-MM activity and with potential synergistic property with bortezomib and lenalidomide, two well established antimyeloma and anti-angiogenic drugs.

\section{MATERIALS AND METHODS}

\section{Patients}

Patients fulfilling the International Myeloma Working Group (IMWG2003 [55]) diagnostic criteria for newly diagnosed MM $(n=47)$ and MGUS $(n=20)$ were studied. The study was approved by the Ethics Committee of the University of Bari "Aldo Moro" Medical School, and all patients provided their informed consent in accordance with the Declaration of Helsinki. 


\section{Reagents}

PP242 and rapamycin were purchased from SigmaAldrich (St Louis, MO), bortezomib from Janssen-Cilag (Cologno Monzese, Italy), and lenalidomide from Celgene Corporation (Milan, Italy). Dulbecco's modified Eagle's medium (DMEM), RPMI 1640, antibiotic/antimycotic, tripsyn/EDTA and phosphate-buffered saline (PBS) without $\mathrm{Ca}^{2+}$ and $\mathrm{Mg}^{2+}$, heat-inactivated fetal bovine serum (FBS) were obtained from Sigma-Aldrich.

\section{ECs isolation and characterization}

Bone marrow aspirates from patients with MGUS and MM were centrifuged on Ficoll- Hypaque (Pharmacia Biotech, Uppsala, Sweden) gradient, and the separated mononuclear cells were left to adhere in complete medium (RPMI-1640 medium supplemented with 10\% FBS). To isolate ECs, BM adherent stromal cells were harvested and incubated with magnetic microbeads coated with anti-CD31 antibody (Miltenyi Biotec, Bergisch Gladbach, Germany) according to manufacturer's instructions. Factor VIII-related antigen, CD31, Vascular Endothelial Growth Factor Receptor 2 (VEGFR2), and Tie2 expression of freshly isolated MGUS-ECs and MM-ECs was verified by flow cytometry FACS Canto II (Becton Dickinson-BD, San Jose, CA, USA).

\section{Western blotting}

Total protein lysate $(35 \mu \mathrm{g})$ from MGUS-ECs and MM-ECs were separated on $4-12 \% \mathrm{NuPAGE}^{\circledR}$ gels (Invitrogen Corp.), electro-transferred to a polyvinylidene difluoride membrane (PerkinElmer Life Science Inc., Boston, MA, USA), and immunoblotted with anti-mTOR, anti-p-mTOR (Ser2448), anti-AKT, anti-p-AKT (Ser473), anti-pS6K1, anti-p-pS6K1 (Thr389), anti-RAPTOR, anti-ERK1/2 (p44/42), anti-p-ERK1/2 (Thr202/Thr204) (Cell Signaling Techology Inc., Danvers, MA, USA), anti-RICTOR (Abcam, Cambridge, UK), and anti- $\beta$ actin antibodies (Sigma-Aldrich). Then, the membranes were incubated with horseradish peroxidase-labeled secondary antibodies (Bio-Rad, Hercules, CA, USA). Immuno-reactive bands were visualized by enhanced chemiluminescence (LiteAblot extend substrate, Euroclone, Pero, Milan, Italy) and the Gel Logic 1,500 Imaging System (Eastman Kodak Co., Rochester, NY, USA), quantified with the Kodak Molecular Imaging Software. The expression bands were quantified as arbitrary optical density (OD) units.

\section{Real time RT- PCR}

Total RNA was isolated using the RNeasy Micro kit (Qiagen, Venlo, Netherlands) and reverse transcripted into total cDNA with the iScript cDNA Synthesis Kit (Bio-Rad). Real time RT-PCR reactions were carried out with the "StepOne Real-Time RT-PCR System" (Applied Biosystems, Waltham, MA, USA), performed with specific primers for mTOR, RAPTOR and RICTOR using Taqman $^{\circledR}$ RT-PCR technology (Applied Biosystems). The gene expression (fold change) was measured with the comparative threshold cycle $(\mathrm{Ct})$ method using glyceraldehyde-3-phosphate dehydrogenase (GAPDH) as endogenous control and the $2^{-\Delta \Delta \mathrm{Ct}}$ formula [56].

\section{RICTOR siRNA transient transfection}

MM-ECs $\left(5 \times 10^{6}\right)$ were transiently transfected for $48 \mathrm{~h}$ and $72 \mathrm{~h}$ with $25 \mathrm{nM}$ and $50 \mathrm{nM}$ of siRNAs specific for RICTOR (target sequences: a) GGGAAUACAACUCCAAAUA; b) GCGAGCUGAUGUAGAAUUA; c) GAAGAUUUA UUGAGUCCUA; d) GACACAAGCACUUCGAUUA) and negative control scramble siRNAs (SMART-pool; Dharmacon RNA Technologies, Lafayette, CO, USA), or with the transfection reagent alone (Lipofectamine, RNAiMAX siRNA transfection reagent, Invitrogen Corp).

\section{Immunofluorescence}

MM-ECs $\left(5 \times 10^{3}\right)$ were cultured on chamber slides (Lab Tek II Chamber Slides, Thermo Scientific Fisher Scientific Inc, Waltham, MA, USA). To demonstrate the colocalization of mTOR with RAPTOR and RICTOR, cells were fixed with $2 \%$ paraformaldehyde, permeabilized with Triton-X100 and then incubated with p-mTOR (Ser2448 immunohistochemistry, Cell Signaling Techology Inc., Danvers, MA, USA), RAPTOR (Merck Millipore, Darmstadt, Germany) and RICTOR (Abcam, Cambridge, UK). Then, cells were incubated with secondary antibodies conjugated with fluorescein isothiocyanate (FITC) and tetramethylrhodamine (TRITC). For F-actin staining, MMECs treated with siRNA and PP242, were fixed with $2 \%$ paraformaldehyde, permeabilized with Triton-X100 and then incubated with phalloidin-FITC (Sigma-Aldrich). Nuclei were counterstained with 4',6-diamidino-2-phenylindole (DAPI) at a concentration of $150 \mathrm{nM}$ for 10 minutes (Invitrogen Corp).

\section{Wound healing assay}

MM-ECs $\left(4 \times 10^{4}\right)$ were grown to confluence on 24-well plates in complete medium. A wound was made by scraping the cell monolayer with a sterile pipette tip. Medium was removed and the cells were cleaned with PBS. Then, they were exposed to serum-free medium (SFM) as negative control, complete DMEM (as positive control) and PP242 at 10, 50, 100 and $200 \mathrm{nM}$. In silencing experiments, the scratch was made at $56 \mathrm{~h}$. After $16 \mathrm{~h}$, cells were fixed and stained. Cell migration was determined by counting the MM-ECs migrated into the "wound", and cells are indicated as the percentage of relative wound closure compared with control. 


\section{Chemotaxis and chemo-invasion assays}

To evaluate the chemotaxis ability of MM-ECs, they were tested $\left(5 \times 10^{4}\right)$ in a Boyden micro-chamber assay toward DMEM alone (negative control), DMEM with $1.5 \%$ FBS added with VEGF and FGF-2 (both at 10 ng/ml; Miltenyi Biotec, Bergisch Gladbach, Germany) as chemo-attractants (positive control) in RICTOR silencing experiments and, in DMEM with $100 \mathrm{nM}$ of PP242 in experiments with the mTOR inhibitor. In the same conditions, the chemoinvasive ability of MM-ECs was evaluated using BD BioCoat ${ }^{\mathrm{TM}}$ Matrigel $^{\mathrm{TM}}$ Invasion Chamber (BD Biosciences, Milan, Italy). After 16h at $37^{\circ} \mathrm{C}$, the migrated/invaded cells were fixed, stained and counted at $400 \mathrm{X}$ on the EVOS inverted microscope (EuroClone, Pero, Milan, Italy).

\section{Adhesion assay}

MM-ECs were treated with $25 \mathrm{nM}$ siRICTOR for $72 \mathrm{~h}$ or with $100 \mathrm{nM}$ PP242 for $48 \mathrm{~h}$, stained with Calcein $\mathrm{AM}$ for $1 \mathrm{~h}$, and then plated $\left(1 \times 10^{3}\right.$ cells per well $)$ in quadruplicate in 96-well fibronectin-coated $(10 \mu \mathrm{g} / \mathrm{mL})$ plate. After 30 minutes, non-adherent cells were washed away and the rate of adherent cells was evaluated reading fluorescence at $495 \mathrm{~nm}$ by VICTOR ${ }^{\text {TM }}$ X3 Multilabel Plate Reader (PerkinElmer Inc., Waltham, MA, USA).

\section{Capillarogenesis assay on matrigel ${ }^{\circledR}$}

MM-ECs pre-transfected with siRICTOR or pretreated with drugs (rapamycin, PP242, lenalidomide, bortezomib) were plated $\left(3.5 \times 10^{4}\right)$ on 48 -well plates coated with Matrigel ${ }^{\circledR}$ (BD Biosciences) in SFM. After $16 \mathrm{~h}$, the skeletonization of the mesh was followed by measurement of mesh areas and vessel length in three randomly chosen fields with the EVOS microscope at $10 \mathrm{X}$.

\section{Apoptosis and proliferation assays}

The apoptotic effect of PP242 was analysed by using the Annexin-V-PE/7aminoactinomycin-D assay (BD) according to manufacturer's instructions. Samples were evaluated by flow cytometry by the FACS Canto II. To determine the effect of PP242 on MM-ECs proliferation, cells were labeled with carboxyfluorescein succinimidyl ester (CFSE) by using CellTrace cell proliferation kit (Molecular Probes Inc.) according to manufacturer's instructions. Labeled MM-ECs $\left(4 \times 10^{4}\right)$ were treated with PP242 at different concentration $(10 \div 200 \mathrm{nM})$ for $72 \mathrm{~h}$, then evaluated by the FACS Canto II.

\section{Zymography}

CM from $5 \times 10^{5}$ MM-ECs, treated or not with $100 \mathrm{nM}$ PP242 for $48 \mathrm{~h}$, were collected, centrifuged at
$450 \mathrm{~g}$ at $4^{\circ} \mathrm{C}$ for 5 minutes to eliminate cell debris, and concentrated to $1 \times 10^{6}$ cells $/ \mathrm{ml}$ at $950 \mathrm{~g}$ at $4^{\circ} \mathrm{C}$ for 60 minutes. The CM were mixed with sodium dodecyl sulphate buffer under non-reducing conditions, and run on Nove ${ }^{\circledR}$ Zymogram gelatine gel (Invitrogen Corp.) at $125 \mathrm{~V}$ for 90 minutes. After electrophoresis, the enzyme was renatured by incubating the gel in Zymogram renaturing buffer containing a non-ionic detergent. The gel was equilibrated in Zymogram developing buffer, and then stained and destained following manufacturer's instructions (Invitrogen Corp.). Regions of protease activity appeared as clear bands against a dark blue background.

\section{Cytokine measurement}

CM by seeding $2 \times 10^{5}$ cells treated or not with PP242 $(100 \mathrm{nM})$ were obtained as reported above. Cytokines were measured by using Q-Plex ${ }^{\mathrm{TM}}$ Array Human Angiogenesis Antigen (Quansys Biosciences, Logan, UT, USA) that detects ANG-2, FGF-2, HGF, IL-8, PDGF-BB, TIMP-1 and $-2, \mathrm{TNF}-\alpha$ and VEGF, according to the manufacturer's instructions. Secreted levels of cytokines were quantified through Q-View Software (Quansys Biosciences).

\section{CAM assay}

Fertilized white Leghorn chicken eggs were incubated at $37^{\circ} \mathrm{C}$ and constant humidity. On day 3, the shell was opened and 2-to-3 $\mathrm{mL}$ of albumen removed to detach the CAM. On day 8, the CAMs were implanted with $1 \mathrm{~mm}^{3}$ sterilized gelatin sponges (Gelfoam Upjohn Co., New York, NY, USA), loaded with SFM alone (negative control), or with conditioned medium (CM) of treated/untreated MM-ECs for $24 \mathrm{~h}$ and $48 \mathrm{~h}$. On day 12 , the angiogenic response was evaluated as the number of vessels converging toward the sponge at $50 \mathrm{X}$ and photographed in ovo by a stereomicroscope (Olympus Italia Srl, Segrate, Milan, Italy).

\section{Matrigel ${ }^{\circledR}$ plug assay}

The anti-angiogenic activity of mTOR inhibitor PP242 was evaluated in vivo in the murine Matrigel ${ }^{\circledR}$ plug assay. Seven-week-old NOD/SCID female mice (Envigo, Bresso, Italy) were injected subcutaneously, into the flank, with $300 \mu \mathrm{L}$ of liquid Matrigel (Cultrex ${ }^{\circledR}$ BME Growth Factor Reduced, Trevigen, Helgerman CT, Gaithersburg) containing 500 ng of FGF-2 and 500 ng of VEGF (Miltenyi Biotec, Bergisch Gladbach, Germany) as positive control, PBS added with CM of MM-ECs treated and not treated with PP242, and PBS alone as negative control. To evaluate PP242 direct effect on the mTOR inhibition, a group of mice injected with proangiogenic cytokines were treated or not intraperitoneally (IP) with PP242 50mg/Kg twice a week [57]. Ten days after injection, mice were sacrificed. Harvested plugs were 
embedded in Tissue Tec Optimal Cutting Temperature (Sakura Finetek USA, Torrance, CA, USA) and analyzed by immunofluorescence microscopy for mouse CD31 expression (Abcam, Cambridge, UK). Animal experiments were approved by local animal ethics committee (OPBA) at the University of Bari "Aldo Moro", and were executed in accordance with national guidelines and regulations.

\section{Statistical analysis}

This was performed using GraphPadPrism5 software. Results were analyzed using the Wilcoxon signed-rank test. $\mathrm{P}<0.03$ was considered statistically significant.

\section{CONFLICTS OF INTEREST}

The authors declare that there are no competing financial interests in relation to this work.

\section{FUNDING}

This work was supported by Associazione Italiana per la Ricerca sul Cancro (AIRC); Investigator Grant (no. 14095 to AV); Special Program Molecular Clinical Oncology 5 per 1000 (no. 9965 to AV), Milan, IT.

\section{REFERENCES}

1. De Raeve HR, Vanderkerken K. The role of the bone marrow microenvironment in multiple myeloma. Histol Histopathol. 2005; 20: 1227-1250.

2. Ria R, Reale A, De Luisi A, Ferrucci A, Moschetta M, Vacca A. Bone marrow angiogenesis and progression in multiple myeloma. Am J Blood Res. 2011; 1: 76-89.

3. Vacca A, Ribatti D, Roncali L, Ranieri G, Serio G, Silvestris F, Dammacco F. Bone marrow angiogenesis and progression in multiple myeloma. Br J Haematol. 1994; 87:503-8.

4. Hose D, Moreaux J, Meissner T, Seckinger A, Goldschmidt H, Benner A, Mahtouk K, Hillengass J, Rème T, De Vos J, Hundemer M, Condomines M, Bertsch U, et al. Induction of angiogenesis by normal and malignant plasma cells. Blood. 2009; 114: 128-143.

5. Roccaro AM, Hideshima T, Raje N, Kumar S, Ishitsuka K, Yasui H, Shiraishi N, Ribatti D, Nico B, Vacca A, Dammacco F, Richardson PG, Anderson KC. Bortezomib mediates antiangiogenesis in multiple myeloma via direct and indirect effects on endothelial cells. Cancer Res. 2006; 1;66:184-9.

6. Knight R. IMiDs: a novel class of immunomodulators. Semin Oncol. 2005; 32:S24-30.

7. De Luisi A, Ferrucci A, Coluccia AM, Ria R, Moschetta M, de Luca E, Pieroni L, Maffia M, Urbani A, Di Pietro G, Guarini A, Ranieri G, Ditonno P, et al. Lenalidomide restrains motility and overangiogenic potential of bone marrow endothelial cells in patients with active multiple myeloma. Clin Cancer Res. 2011; 17: 1935-1946.

8. Sarbassov DD, Ali SM, Sabatini DM. Growing roles for the mTOR pathway. Curr Opin Cell Biol. 2005; 17: 596-603.

9. Nandagopal N, Roux PP. Regulation of global and specific mRNA translation by the mTOR signaling pathway. Translation (Austin). 2015; 3: e983402.

10. Dunlop EA, Tee AR. mTOR and autophagy: a dynamic relationship governed by nutrients and energy. Semin Cell Dev Biol. 2014; 36:121-9.

11. Jacinto E, Loewith R, Schmidt A, Li S, Rüegg MA, Hall A, Hall MN. Mammalian TOR complex 2 controls the actin cytoskeleton and is rapamycin insensitive. Nature Cell Biology. 2004; 6: 1122-1128.

12. Wang S, Amato KR, Song W, Youngblood V, Lee K, Boothby M, Brantley-Sieders DM, Chen J. Regulation of endothelial cell proliferation and vascular assembly through distinct mTORC2 signaling pathways. Mol Cell Biol. 2015; 35:1299-313.

13. Sarbassov DD, Guertin DA, Ali SM, Sabatini DM. Phosphorylation and regulation of $\mathrm{Akt} / \mathrm{PKB}$ by the rictormTOR complex. Science. 2005; 307:1098-1101.

14. Zhou H, Huang S. Role of mTOR signaling in tumor cell motility, invasion and metastasis. Curr Protein Pept Sci. $2011 ; 12: 30-42$.

15. Shi Y, Gera J, Hu L, Hsu J, Bookstein R, Bookstein R, Li W, Lichtenstein A. Enhanced sensitivity of multiple myeloma cells containing PTEN mutations to CCI-779. Cancer Res. 2002; 62: 5027-5034.

16. Strömberg T, Dimberg A, Hammarberg A, Carlson K, Osterborg A, Nilsson K, Jernberg-Wiklund H. Rapamycin sensitizes multiple myeloma cells to apoptosis induced by dexamethasone. Blood. 2004; 103: 3138-3147.

17. Raje N, Kumar S, Hideshima T, Ishitsuka K, Chauhan D, Mitsiades C Podar K, Le Gouill S, Richardson P, Munshi NC, Stirling DI, Antin JH, Anderson KC. Combination of the mTOR inhibitor rapamycin and CC-5013 has synergistic activity in multiple myeloma. Blood. 2004; 104: 4188-4193.

18. Raje N, Richardson P, Hari PN, Mahindra A, Kaster S, Connolly C, Connolly C, Rivera L, Chetri G, Dean S, Jacobs P, Saad A, Laubach J, et al. An open label phase I study of the safety and efficacy of RAD001 in combination with lenalidomide in the treatment of patients with relapsed and refractory multiple myeloma. Blood. 2009; 114: 1483.

19. Ghobrial IM, Munshi N, Schlossman R, Chuma S, Leduc R, Nelson M, Sam A, O'Connor K, Harris B, Warren D, Dollard AM, Laubach J, Vij R, et al. Phase I trial of CCI-779 (Temsirolimus) and weekly bortezomib in relapsed and/or refractory multiple myeloma. Blood. 2008; 112: 1266.

20. Chen XG, Liu F, Song XF, Wang ZH, Dong ZQ, Hu ZQ, Lan RZ, Guan W, Zhou TG, Xu XM, Lei H, Ye $Z Q$, Peng EJ, et al. Rapamycin regulates Akt and ERK phosphorylation through mTORC1 and mTORC2 signaling pathways. Mol Carcinog. 2010; 49: 603-610. 
21. Maiso P, Liu Y, Morgan B, Azab AK, Ren P, Martin MB, Zhang Y, Liu Y, Sacco A, Ngo H, Azab F, Quang P, Rodig SJ, et al. Defining the role of TORC1/2 in multiple myeloma. Blood. 2011; 118: 6860-6870.

22. Zhou HY, Huang SL. Current development of the second generation of mTOR inhibitors as anticancer agents. Chin J Cancer. 2012; 31: 8-18.

23. Apsel B, Blair JA, Gonzalez B, Nazif TM, Feldman ME, Aizenstein B, Hoffman R, Williams RL, Shokat KM, Knight ZA. Targeted polypharmacology: discovery of dual inhibitors of tyrosine and phosphoinositide kinases. Nat Chem Biol. 2008; 4: 691-699.

24. Feldman ME, Apsel B, Uotila A, Loewith R, Knight ZA, Ruggero D, Shokat KM. Active-site inhibitors of mTOR target rapamycin-resistant outputs of mTORC1 and mTORC2. PLoS Biol. 2009; 7: e1000038.

25. Hoang B, Frost P, Shi Y, Belanger E, Benavides A, Pezeshkpour G, Cappia S, Guglielmelli T, Gera J, Lichtenstein A. Targeting TORC2 in multiple myeloma with a new mTOR kinase inhibitor. Blood. 2010; 116: 4560-4568.

26. Falcon BL, Barr S, Gokhale PC, Chou J, Fogarty J, Depeille P, Miglarese M, Epstein DM, McDonald DM. Reduced VEGF Production, Angiogenesis, and Vascular Regrowth Contribute to the Antitumor Properties of Dual mTORC1/ mTORC2 Inhibitors. Cancer Res. 2011; 71: 1573-1583.

27. Lee HP, Lin CY, Shih JS, Fong YC, Wang SW, Li TM, Tang CH. Adiponectin promotes VEGF-A-dependent angiogenesis in human chondrosarcoma through PI3K, Akt, mTOR, and HIF- $\alpha$ pathway. Oncotarget. 2015; 6: 3674636761. https://doi.org/10.18632/oncotarget.5479.

28. Dodd KM, Yang J, Shen MH, Sampson JR, Tee AR. mTORC1 drives HIF-1 $\alpha$ and VEGF-A signalling via multiple mechanisms involving 4E-BP1, S6K1 and STAT3. Oncogene. 2015; 34: 2239-2250.

29. Sun S, Chen S, Liu F, Wu H, McHugh J, Bergin IL, Gupta A, Adams D, Guan JL. Constitutive Activation of mTORC1 in Endothelial Cells Leads to the Development and Progression of Lymphangiosarcoma through VEGF Autocrine Signaling. Cancer Cell. 2015; 28: 758-772.

30. Frost P, Berlanger E, Mysore V, Hoang B, Shi Y, Gera J, Lichtenstein A. Mammalian Target of Rapamycin Inhibitors Induce Tumor Cell Apoptosis In Vivo Primarily by Inhibiting VEGF Expression and Angiogenesis. Journal of Oncology. 2013; 2013:897025.

31. Sarbassov DD, Ali SM, Kim DH, Guertin DA, Latek RR, Erdjument-Bromage H, Tempst P, Sabatini DM. Rictor, a novel binding partner of $\mathrm{mTOR}$, defines a rapamycininsensitive and raptor- independent pathway that regulates the cytoskeleton. Curr Biol. 2004; 14: 1296-1302.

32. Kim EK, Yun SJ, Ha JM, Kim YW, Jin IH, Yun J, Shin HK, Song SH, Kim JH, Lee JS, Kim CD, Bae SS. Selective activation of Akt1 by mammalian target of rapamycin complex 2 regulates cancer cell migration, invasion, and metastasis. Oncogene. 2011; 30: 2954-2963. https://doi. org/10.1038/onc.2011.22.

33. Wo lpin BM, Hezel AF, Abrams T, Blaszkowsky LS, Meyerhardt JA, Chan JA, Enzinger PC, Allen B, Clark JW, Ryan DP, Fuchs CS. Oral mTOR Inhibitor Everolimus in Patients With Gemcitabine-Refractory Metastatic Pancreatic Cancer. Journal of Clinical Oncology. 2009; 27:193-198.

34. Wolpin BM, Ng K, Zhu AX, Abrams T, Enzinger PC, McCleary NJ, Schrag D, Kwak EL, Allen JN, Bhargava P, Chan JA, Goessling W, Blaszkowsky LS, et al. Multicenter Phase II Study of Tivozanib (AV-951) and Everolimus (RAD001) for Patients With Refractory, Metastatic Colorectal Cancer. The Oncologist. 2013; 18:377-378.

35. Morrow PK, Wulf GM, Ensor J, Booser DJ, Moore JA, Flores PR, Xiong Y, Zhang S, Krop IE, Winer EP, Kindelberger DW, Coviello J, Sahin AA, et al. Phase I/II Study of Trastuzumab in Combination With Everolimus (RAD001) in Patients With HER2-Overexpressing Metastatic Breast Cancer Who Progressed on TrastuzumabBased Therapy. Journal of Clinical Oncology. 2011; 29:3126-3132.

36. Price KA, Azzoli CG, Krug LM, Pietanza MC, Rizvi NA, Pao W, Kris MG, Riely GJ, Heelan RT, Arcila ME, Miller VA. Phase II Trial of Gefitinib and Everolimus in Advanced Non-small Cell Lung Cancer. J Thorac Oncol. 2010; 5:16231629. https://doi.org/10.1097/JTO.0b013e3181ec1531.

37. Bracho-Valdés I, Moreno-Alvarez P, Valencia-Martínez I, Robles-Molina E, Chávez-Vargas L, Vázquez-Prado J. mTORC1- and mTORC2-interacting proteins keep their multifunctional partners focused. IUBMB Life. 2011; 63: 896-914.

38. Ghobrial IM, Siegel DS, Vij R, Berdeja JG, Richardson PG, Neuwirth R, Patel CG, Zohren F, Wolf JL. TAK-228 (formerly MLN0128), an investigational oral dual TORC1/2 inhibitor: A phase I dose escalation study in patients with relapsed or refractory multiple myeloma, non-Hodgkin lymphoma, or Waldenström's macroglobulinemia. Am J Hematol. 2016; 91: 400-405.

39. Calimeri T, Ferreri AJM. m-TOR inhibitors and their potential role in haematological malignancies. $\mathrm{Br} \mathrm{J}$ Haematol. 2017; 177: 684-702.

40. Parri M, Chiarugi P. Rac and Rho GTPases in cancer cell motility control. Cell Commun Signal. 2010; 8:23.

41. Lamalice L, Le Boeuf F, Huot J. Endothelial Cell Migration During Angiogenesis. Circ Res. 2007; 30;100:782-94.

42. Ria R, Vacca A, Ribatti D, Di Raimondo F, Merchionne F, Dammacco F. Alpha(v)beta(3) integrin engagement enhances cell invasiveness in human multiple myeloma. Haematologica. 2002; 87: 836-845.

43. Weis SM, Cheresh DA. av Integrins in Angiogenesis and Cancer. Cold Spring Harbor Perspectives in Medicine. 2011; 1:a006478.

44. Chantaravisoot N, Wongkongkathep P, Loo JA, Mische PS, Tamanoi F. Significance of filamin A in mTORC2 function in glioblastoma. Molecular Cancer. 2015; 14:127. 
45. Karar J, Maity A. PI3K/AKT/mTOR Pathway in Angiogenesis. Front Mol Neurosci. 2011; 4:51.

46. Chen JS, Wang Q, Fu XH, Huang XH, Chen XL, Cao LQ, Chen LZ, Tan HX, Li W, Bi J, Zhang LJ. Involvement of PI3K/PTEN/AKT/mTOR pathway in invasion and metastasis in hepatocellular carcinoma: Association with MMP-9. Hepatology Research. 2009; 39:177-186.

47. Ria R, Vacca A, Russo F, Cirulli T, Massaia M, Tosi P, Cavo M, Guidolin D, Ribatti D, Dammacco F. A VEGF-dependent autocrine loop mediates proliferation and capillarogenesis in bone marrow endothelial cells of patients with multiple myeloma. Thromb Haemost. 2004; 92: 1438-1445.

48. Ferrucci A, Moschetta M, Frassanito MA, Berardi S, Catacchio I, Ria R, Racanelli V, Caivano A, Solimando AG, Vergara D, Maffia M, Latorre D, Rizzello A, et al. A HGF/ cMET autocrine loop is operative in multiple myeloma bone marrow endothelial cells and may represent a novel therapeutic target. Clin Cancer Res. 2014; 20: 5796-5807.

49. Asahara T, Chen D, Takahashi T, Fujikawa K, Kearney M, Magner M, Yancopoulos GD, Isner JM. Tie2 receptor ligands, angiopoietin-1 and angiopoietin-2, modulate VEGF-induced postnatal neovascularization. Circ Res. 1998; 10: 83:233-40.

50. Lobov IB, Brooks PC, Lang RA. Angiopoietin-2 displays VEGF-dependent modulation of capillary structure and endothelial cell survival in vivo. Proceedings of the National Academy of Sciences of the United States of America. 2002; 99:11205-11210.

51. Belloni D, Marcatti M, Ponzoni M, Ciceri F, Veschini L, Corti A, Caligaris Cappio F, Ferrarini M, Ferrero E. Angiopoietin-2 in Bone Marrow milieu promotes Multiple
Myeloma-associated angiogenesis. Exp Cell Res. 2015; 330: 1-12.

52. Ria R, Catacchio I, Berardi S, De Luisi A, Caivano A, Piccoli C, Ruggieri V, Frassanito MA, Ribatti D, Nico B, Annese T, Ruggieri S, Guarini A, et al. HIF-1 $\alpha$ of bone marrow endothelial cells implie relapse and drug resistance in patients with multiple myeloma and may act as a therapeutic target. Clin Cancer Res. 2014; 20: 847-858.

53. Moschetta M, Basile A, Ferrucci A, Frassanito MA, Rao L, Ria R, Solimando AG, Giuliani N, Boccarelli A, Fumarola F, Coluccia M, Rossini B, Ruggieri S, et al. Novel targeting of phospho-cMet overcomes drug resistance and induces antitumor activity in multiple myeloma. Clin Cancer Res. 2013; 19: 4371-4382.

54. Vacca A, Scavelli C, Montefusco V, Di Pietro G, Neri A, Mattioli M, Bicciato S, Nico B, Ribatti D, Dammacco F, Corradini P. Thalidomide downregulates angiogenic genes in bone marrow endothelial cells of patients with active multiple myeloma. J Clin Oncol. 2005; 23: 5334-46.

55. International Myeloma Working Group. Criteria for the classification of monoclonal gammopathies, multiple myeloma and related disorders: a report of the International Myeloma Working Group. Br J Haematol. 2003; 121: 749-57.

56. Livak KJ, Schmittgen TD. Analysis of relative gene expression data using real-time quantitative PCR and the 2(-Delta Delta C(T)) Method. Methods. 2001; 25: 402-408.

57. Cheng L, Xia Z, Bian X, Li G, Hu J, Cao Y, Wang Q, Qian X. Combination of cetuximab and PP242 synergistically suppress the progression of wild-type KRAS colorectal carcinoma. Onco Targets Ther. 2015; 8: 3185-3192. 